Proceedings of the Institute of Mathematics and Mechanics,

National Academy of Sciences of Azerbaijan

Volume 46, Number 1, 2020, Pages 3-15

https://doi.org/10.29228/proc.13

\title{
ON THE GENERALIZED MIXED SCHWARZ INEQUALITY
}

\author{
MOHAMMAD W. ALOMARI
}

\begin{abstract}
In this work, an extension of the generalized mixed Schwarz inequality is proved. A companion of the generalized mixed Schwarz inequality is established by merging both Cartesian and Polar decompositions of operators. Based on that some numerical radius inequalities are proved.
\end{abstract}

\section{Introduction}

Let $\mathscr{B}(\mathscr{H})$ be the Banach algebra of all bounded linear operators defined on a complex Hilbert space $(\mathscr{H} ;\langle\cdot, \cdot\rangle)$ with the identity operator $1_{\mathscr{H}}$ in $\mathscr{B}(\mathscr{H})$. A bounded linear operator $A$ defined on $\mathscr{H}$ is selfadjoint if and only if $\langle A x, x\rangle \in \mathbb{R}$ for all $x \in \mathscr{H}$. Consider the real vector space $\mathscr{B}(\mathscr{H})_{s a}$ of self-adjoint operators on $\mathscr{H}$ and its positive cone $\mathscr{B}(\mathscr{H})^{+}$of positive operators on $\mathscr{H}$. A partial order is naturally equipped on $\mathscr{B}(\mathscr{H})_{s a}$ by defining $A \leq B$ if and only if $B-A \in \mathscr{B}(\mathscr{H})^{+}$. We write $A>0$ to mean that $A$ is a strictly positive operator, or equivalently, $A \geq 0$ and $A$ is invertible. When $\mathscr{H}=\mathbb{C}^{n}$, we identify $\mathscr{B}(\mathscr{H})$ with the algebra $\mathfrak{M}_{n \times n}$ of $n$-by- $n$ complex matrices. Then, $\mathfrak{M}_{n \times n}^{+}$is just the cone of $n$-by- $n$ positive semidefinite matrices.

The Schwarz inequality for positive operators reads that if $A$ is a positive operator in $\mathscr{B}(\mathscr{H})$, then

$$
|\langle A x, y\rangle|^{2} \leq\langle A x, x\rangle\langle A y, y\rangle
$$

for any vectors $x, y \in \mathscr{H}$.

In 1951, Reid [16] proved an inequality which in some senses considered a variant of the Schwarz inequality. In fact, he proved that for all operators $A \in$ $\mathscr{B}(\mathscr{H})$ such that $A$ is positive and $A B$ is selfadjoint then

$$
|\langle A B x, y\rangle| \leq\|B\|\langle A x, x\rangle,
$$

for all $x \in \mathscr{H}$. In [5], Halmos presented his stronger version of the Reid inequality (1.2) by replacing $r(B)$ instead of $\|B\|$.

In 1952, Kato [13] introduced a companion inequality of (1.1), called the mixed Schwarz inequality, which asserts

$$
|\langle A x, y\rangle|^{2} \leq\left\langle|A|^{2 \alpha} x, x\right\rangle\left\langle\left|A^{*}\right|^{2(1-\alpha)} y, y\right\rangle, \quad 0 \leq \alpha \leq 1 .
$$

2010 Mathematics Subject Classification. Primary: 47A30, 47A12 Secondary: 15A60, 47A63.

Key words and phrases. Mixed Schwarz inequality, Numerical radius, norm inequalities, Reid inequality. 
for every operators $A \in \mathscr{B}(\mathscr{H})$ and any vectors $x, y \in \mathscr{H}$, where $|A|=\left(A^{*} A\right)^{1 / 2}$.

In 1988, Kittaneh [12] proved a very interesting extension combining both the Halmos-Reid inequality (1.2) and the mixed Schwarz inequality (1.3). His result reads that

$$
|\langle A B x, y\rangle| \leq r(B)\|f(|A|) x\|\left\|g\left(\left|A^{*}\right|\right) y\right\|
$$

for any vectors $x, y \in \mathscr{H}$, where $A, B \in \mathscr{B}(\mathscr{H})$ such that $|A| B=B^{*}|A|$ and $f, g$ are nonnegative continuous functions defined on $[0, \infty)$ satisfying that $f(t) g(t)=t$ $(t \geq 0)$. Clearly, choose $f(t)=t^{\alpha}$ and $g(t)=t^{1-\alpha}$ with $B=1_{\mathscr{H}}$ we refer to (1.3). Moreover, choosing $\alpha=\frac{1}{2}$ some manipulations refer to the Halmos version of the Reid inequality.

In 2006, Lin and Dragomir [14] proved the following sequence of inequalities of Halmos-Ried's type:

$$
\left.\begin{array}{l}
|\langle T x, y\rangle|^{2} \leq r(T)\langle T x, x\rangle\|y\|^{2} \\
|\langle T S x, C y\rangle| \leq r(S) r(C)\langle T x, x\rangle^{1 / 2}\langle T y, y\rangle^{1 / 2} \\
|\langle T S x, C y\rangle| \leq r(S) r(C)\langle T x, x\rangle \\
|\langle A x, B y\rangle|^{2} \leq r(A) r(B)\|A x\|\|B y\|\|x\|\|y\|
\end{array}\right\}
$$

where $A, B, C, T, S \in \mathscr{B}(\mathscr{H})$ such that $T$ is non-negative operator, $S$ and $C$ are arbitrary operators, and $T S, T C, A$ and $B$ be selfadjoint operators, for all vectors $x, y \in \mathscr{H}$.

For a bounded linear operator $T$ on a Hilbert space $\mathscr{H}$, the numerical range $W(T)$ is the image of the unit sphere of $\mathscr{H}$ under the quadratic form $x \rightarrow\langle T x, x\rangle$ associated with the operator. More precisely,

$$
W(T)=\{\langle T x, x\rangle: x \in \mathscr{H},\|x\|=1\}
$$

Also, the numerical radius is defined to be

$$
w(T)=\sup \{|\lambda|: \lambda \in W(T)\}=\sup _{\|x\|=1}|\langle T x, x\rangle| .
$$

The spectral radius of an operator $T$ is defined to be

$$
r(T)=\sup \{|\lambda|: \lambda \in \operatorname{sp}(T)\} .
$$

We recall that, the usual operator norm of an operator $T$ is defined to be

$$
\|T\|=\sup \{\|T x\|: x \in H,\|x\|=1\} .
$$

It is well known that $w(\cdot)$ defines an operator norm on $\mathscr{B}(\mathscr{H})$ which is equivalent to operator norm $\|\cdot\|$. Moreover, we have

$$
\frac{1}{2}\|T\| \leq w(T) \leq\|T\|
$$

for any $T \in \mathscr{B}(\mathscr{H})$ and this inequality is sharp.

In 2003, Kittaneh [9] refined the right-hand side of (1.6), where he proved that

$$
w(T) \leq \frac{1}{2}\left(\|T\|+\left\|T^{2}\right\|^{1 / 2}\right)
$$


for any $T \in \mathscr{B}(\mathscr{H})$.

After that in 2005, the same author in [7] proved that

$$
\frac{1}{4}\left\|A^{*} A+A A^{*}\right\| \leq w^{2}(A) \leq \frac{1}{2}\left\|A^{*} A+A A^{*}\right\| .
$$

The inequality is sharp. This inequality was also reformulated and generalized in [3] but in terms of Cartesian decomposition.

In 2007, Yamazaki [18] improved (1.7) by proving that

$$
w(T) \leq \frac{1}{2}(\|T\|+w(\widetilde{T})) \leq \frac{1}{2}\left(\|T\|+\left\|T^{2}\right\|^{1 / 2}\right)
$$

where $\widetilde{T}=|T|^{1 / 2} U|T|^{1 / 2}$ and $U$ is the unitary operator in the polar decomposition $T$ of the form $T=U|T|$.

In 2008, Dragomir [2] used Buzano inequality to improve (1.1), where he proved that

$$
w^{2}(T) \leq \frac{1}{2}\left(\|T\|+w\left(T^{2}\right)\right)
$$

This result was also recently generalized by Sattari et al. in [17]. For more recent results about the numerical radius see [1], [8], [10], [15] and the recent monograph study [1]. For basic properties of numerical radius and other related topics the reader may refer to the classical books of Gustafson \& Rao [4] and Horn \& Johnson [6].

In this work, an extension of the Kittaneh inequality (1.4) and a generalization of Lin-Dragomir version of Halmos-Ried type inequalities are proved. Namely, we generalize the Kittaneh inequality (1.4) which already extend the mixed Schwarz inequality (1.3) to be in more general case. A generalization of the obtained result for several operators is also pointed out. A companion of the generalized mixed Schwarz inequality (or Kittaneh inequality) in which the Cartesian decomposition of operators is replaced by the polar decomposition is also given. As application, some numerical radius and norm inequalities are established.

\section{The Result(s)}

This section is divided into two parts; the first part is devoted to generalize the Kittaneh inequality (1.4) with other related consequences. In the second part, by merging the Cartesian and Polar decompositions of operators, we present a new type of mixed Schwarz inequality called "the Mixed hybrid Schwarz inequality".

2.1. The mixed Schwarz inequality. Let us start with the following elementary result which is a simple consequence of (1.3).

Lemma 2.1. Let $A, C, D \in \mathscr{B}(\mathscr{H})$ such that $|A| D=D^{*}|A|$ and $\left|A^{*}\right| C=C^{*}\left|A^{*}\right|$. If $f$ and $g$ are nonnegative continuous functions on $[0, \infty)$ satisfying $f(t) g(t)=t$ $(t \geq 0)$, then

$$
|\langle A D u, C v\rangle|^{2} \leq\left\langle D^{*} f^{2}(|A|) D u, u\right\rangle\left\langle C^{*} g^{2}\left(\left|A^{*}\right|\right) C v, v\right\rangle
$$

for all vectors $u, v \in \mathscr{H}$. 
Proof. Since $x, y \in \mathscr{H}$ are arbitrary vectors, by the given assumptions there exists $u, v \in \mathscr{H}$ respectively; such that $x=D u$ and $y=C v$, and this is true for any $x, y \in \mathscr{H}$. Therefore by setting $B=1_{\mathscr{H}}$ in (1.4). Then we have

$$
\begin{aligned}
|\langle A D u, C v\rangle|^{2} & \leq\|f(|A|) D u\|^{2}\left\|g\left(\left|A^{*}\right|\right) C v\right\|^{2} \\
& \leq\langle f(|A|) D u, f(|A|) D u\rangle\left\langle g\left(\left|A^{*}\right|\right) C v, g\left(\left|A^{*}\right|\right) C v\right\rangle \\
& \leq\left\langle D^{*} f^{2}(|A|) D u, u\right\rangle\left\langle C^{*} g^{2}\left(\left|A^{*}\right|\right) C v, v\right\rangle .
\end{aligned}
$$

For all vectors $u, v \in \mathscr{H}$, which proves the result.

Remark 2.1. Let $A, C, D \in \mathscr{B}(\mathscr{H})$ be as in Lemma 2.1. In particular case, choosing $f(t)=t^{\alpha}, g(t)=t^{1-\alpha}, t \geq 0$ in (2.1) we get

$$
|\langle A D u, C v\rangle|^{2} \leq\left\langle D^{*}|A|^{2 \alpha} D u, u\right\rangle\left\langle C^{*}\left|A^{*}\right|^{2(1-\alpha)} C v, v\right\rangle .
$$

In special case for $\alpha=\frac{1}{2}$ we have

$$
|\langle A B u, C v\rangle|^{2} \leq\left\langle D^{*}|A| D u, u\right\rangle\left\langle C^{*}\left|A^{*}\right| C v, v\right\rangle
$$

for all vectors $u, v \in \mathscr{H}$.

Now, we are ready to present our generalization of (1.4) and the above inequality (2.1) for any bounded linear operators.

Corollary 2.1. Let $A, B, C \in \mathscr{B}(\mathscr{H})$ such that $|A| B=B^{*}|A|$ and $\left|A^{*}\right| C=$ $C^{*}\left|A^{*}\right|$. If $f$ and $g$ are nonnegative continuous functions on $[0, \infty)$ satisfying $f(t) g(t)=t(t \geq 0)$, then

$$
|\langle A B x, C u\rangle| \leq r(B) r(C)\|f(|A|) x\|\left\|g\left(\left|A^{*}\right|\right) u\right\|
$$

for all vectors $x, u \in \mathscr{H}$.

Proof. It's enough to show that the inequality

$$
\begin{aligned}
|\langle A B x, C u\rangle|^{2^{n} \leq} \leq & \left\langle f^{2}(|A|) B^{2^{n}} x, x\right\rangle\left\langle f^{2}(|A|) x, x\right\rangle^{2^{n-1}-1} \\
& \times\left\langle g^{2}\left(\left|A^{*}\right|\right) C^{2^{n}} u, u\right\rangle\left\langle g^{2}\left(\left|A^{*}\right|\right) u, u\right\rangle^{2^{n-1}-1},
\end{aligned}
$$

is valid for all positive integer $n$. The proof is going likewise the proof of Theorem 5 in [12] taking into account Lemma 2.1.

Corollary 2.2. Let $A, C \in \mathscr{B}(\mathscr{H})$ such that $\left|A^{*}\right| C=C^{*}\left|A^{*}\right|$. If $f$ and $g$ as in Corollary 2.1, then

$$
|\langle A x, C u\rangle| \leq r(C)\|f(|A|) x\|\left\|g\left(\left|A^{*}\right|\right) u\right\|
$$

for any vectors $x, u \in \mathscr{H}$.

Proof. Setting $B=1_{\mathscr{H}}$ in (2.2) we get the required result.

Corollary 2.3. Let $A, B, C \in \mathscr{B}(\mathscr{H})$ such that $|A| B=B^{*}|A|$ and $\left|A^{*}\right| C=$ $C^{*}\left|A^{*}\right|$. Then

$$
|\langle A B x, C u\rangle|^{2} \leq r^{2}(B) r^{2}(C)\left\langle|A|^{2 \alpha} x, x\right\rangle\left\langle\left|A^{*}\right|^{2(1-\alpha)} u, u\right\rangle
$$

for any vectors $x, u \in \mathscr{H}$. In particular we have

$$
|\langle B x, C u\rangle| \leq r(B) r(C)\langle x, x\rangle\langle u, u\rangle .
$$


Proof. Setting $f(t)=t^{\alpha}$ and $g(t)=t^{1-\alpha}, 0 \leq \alpha \leq 1, t \geq 0$ in Corollary 2.1. The particular case follows by setting $A=1_{\mathscr{H}}$ in (2.4).

A more general mixed Schwarz inequality can be stated as follows:

Corollary 2.4. Let $A, D, B_{1}, B_{2}, C_{1}, C_{2} \in \mathscr{B}(\mathscr{H})$ such that

$$
\begin{aligned}
& |A| B_{1}=B_{1}^{*}|A| \quad \text { and } \quad\left|A^{*}\right| C_{1}=C_{1}^{*}\left|A^{*}\right|, \\
& |D| B_{2}=B_{2}^{*}|D| \quad \text { and } \quad\left|D^{*}\right| C_{2}=C_{2}^{*}\left|D^{*}\right| \text {. }
\end{aligned}
$$

If $f$ and $g$ are nonnegative continuous functions on $[0, \infty)$ satisfying $f(t) g(t)=t$ $(t \geq 0)$, then

$$
\begin{aligned}
& \quad\left|\left\langle\left(C_{1}^{*} A B_{1}+C_{2}^{*} D B_{2}\right) x, u\right\rangle\right| \\
& \leq r\left(B_{1}\right) r\left(C_{1}\right)\|f(|A|) x\|\left\|g\left(\left|A^{*}\right|\right) u\right\| \\
& \quad+r\left(B_{2}\right) r\left(C_{2}\right)\|f(|D|) x\|\left\|g\left(\left|D^{*}\right|\right) u\right\| \\
& \leq \max \left\{r\left(B_{1}\right) r\left(C_{1}\right), r\left(B_{2}\right) r\left(C_{2}\right)\right\} \cdot\left(\|f(|A|) x\|^{p}+\|f(|D|) x\|^{p}\right)^{1 / p} \\
& \quad \times\left(\left\|g\left(\left|A^{*}\right|\right) u\right\|^{q}+\left\|g\left(\left|D^{*}\right|\right) u\right\|^{q}\right)^{1 / q}
\end{aligned}
$$

for any vectors $x, u \in \mathscr{H}$ and all $p>1$ with $q=\frac{p}{p-1}$.

Proof. Since

$$
\begin{aligned}
\left|\left\langle\left(C_{1}^{*} A B_{1}+C_{2}^{*} D B_{2}\right) x, u\right\rangle\right| & =\left|\left\langle C_{1}^{*} A B_{1} x, u\right\rangle+\left\langle C_{2}^{*} D B_{2} x, u\right\rangle\right| \\
& \leq\left|\left\langle C_{1}^{*} A B_{1} x, u\right\rangle\right|+\left|\left\langle C_{2}^{*} D B_{2} x, u\right\rangle\right| .
\end{aligned}
$$

So that the first inequality follows from (2.2). The second inequality follows by applying the Hölder inequality.

Corollary 2.5. Let $A, D, B_{1}, B_{2}, C_{1}, C_{2} \in \mathscr{B}(\mathscr{H})$ such that

$$
\begin{aligned}
& |A| B_{1}=B_{1}^{*}|A| \quad \text { and } \quad\left|A^{*}\right| C_{1}=C_{1}^{*}\left|A^{*}\right| \text {, } \\
& |D| B_{2}=B_{2}^{*}|D| \quad \text { and } \quad\left|D^{*}\right| C_{2}=C_{2}^{*}\left|D^{*}\right| \text {. }
\end{aligned}
$$

If $f$ and $g$ are nonnegative continuous functions on $[0, \infty)$ satisfying $f(t) g(t)=t$ $(t \geq 0)$, then

$$
\begin{aligned}
& \left|\left\langle\left(C_{1}^{*} A B_{1}+C_{2}^{*} D B_{2}\right) x, u\right\rangle\right|^{2} \\
& \leq r^{2}\left(B_{1}\right) r^{2}\left(C_{1}\right)\left\langle|A|^{2 \alpha} x, x\right\rangle\left\langle\left|A^{*}\right|^{2(1-\alpha)} u, u\right\rangle \\
& \quad+r^{2}\left(B_{2}\right) r^{2}\left(C_{2}\right)\left\langle|D|^{2 \alpha} x, x\right\rangle\left\langle\left|D^{*}\right|^{2(1-\alpha)} u, u\right\rangle
\end{aligned}
$$

for any vectors $x, u \in \mathscr{H}$.

Proof. Setting $f(t)=t^{\alpha}$ and $g(t)=t^{1-\alpha}, 0 \leq \alpha \leq 1, t \geq 0$ in Corollary 2.4.

In fact, one may establish a generalization of Corollary 2.4 to several operators, by letting $A_{i}, B_{i}, C_{i} \in \mathscr{B}(\mathscr{H})(i=1, \cdots, n)$ such that

$$
\left|A_{i}\right| B_{i}=B_{i}^{*}\left|A_{i}\right| \quad \text { and } \quad\left|A_{i}^{*}\right| C_{i}=C_{i}^{*}\left|A_{i}^{*}\right| \text {. }
$$


If $f, g$ are as above, proceeding as in the presented proof above, then we have

$$
\begin{aligned}
& \left|\left\langle\left(\sum_{i=1}^{n} C_{i}^{*} A_{i} B_{i}\right) x, u\right\rangle\right| \\
& \leq \sum_{i=1}^{n} r\left(B_{i}\right) r\left(C_{i}\right)\left\|f\left(\left|A_{i}\right|\right) x\right\|\left\|g\left(\left|A_{i}^{*}\right|\right) u\right\| \\
& \leq \max _{1 \leq i \leq n}\left\{r\left(B_{i}\right) r\left(C_{i}\right)\right\} \cdot\left(\sum_{i=1}^{n}\left\|f\left(\left|A_{i}\right|\right) x\right\|^{p}\right)^{1 / p}\left(\sum_{i=1}^{n}\left\|g\left(\left|A_{i}^{*}\right|\right) u\right\|^{q}\right)^{1 / q} .
\end{aligned}
$$

For all $x, u \in \mathscr{H}$, which follows by the properties of ' $m a x$ ' and Hölder inequality, where $p, q$ are conjugate exponents, i.e., $p, q>1$ with $\frac{1}{p}+\frac{1}{q}=1$.

Thus, one may has the following norm inequality

$$
\begin{aligned}
& \left\|\sum_{i=1}^{n} C_{i}^{*} A_{i} B_{i}\right\| \\
& \quad \leq \max _{1 \leq i \leq n}\left\{r\left(B_{i}\right) r\left(C_{i}\right)\right\} \cdot\left(\sum_{i=1}^{n}\left\|f\left(\left|A_{i}\right|\right)\right\|^{p}\right)^{1 / p}\left(\sum_{i=1}^{n}\left\|g\left(\left|A_{i}^{*}\right|\right)\right\|^{q}\right)^{1 / q} .
\end{aligned}
$$

For instance, consider $f(t)=t^{\alpha}$ and $g(t)=t^{1-\alpha}$, one has from (2.8) that

$$
\left\|\sum_{i=1}^{n} C_{i}^{*} A_{i} B_{i}\right\| \leq \max _{1 \leq i \leq n}\left\{r\left(B_{i}\right) r\left(C_{i}\right)\right\} \cdot\left(\sum_{i=1}^{n}\left\|\left|A_{i}\right|^{\alpha}\right\|^{p}\right)^{1 / p}\left(\sum_{i=1}^{n}\left\|\left|A_{i}^{*}\right|^{1-\alpha}\right\|^{q}\right)^{1 / q} .
$$

Also, if $C_{i}=B_{i}=1_{\mathscr{H}}$ for all $i=1, \cdots, n$, then the last inequality reduces to

$$
\left\|\sum_{i=1}^{n} A_{i}\right\| \leq\left(\sum_{i=1}^{n}\left\|\left|A_{i}\right|^{\alpha}\right\|^{p}\right)^{1 / p}\left(\sum_{i=1}^{n}\left\|\left|A_{i}^{*}\right|^{1-\alpha}\right\|^{q}\right)^{1 / q} \text {. }
$$

2.2. The mixed hybrid Schwarz inequality. Merging both Cartesian and Polar decompositions of operators will produce a new hybrid Mixed Schwarz inequality including both decompositions. The next result provides a new extension of the mixed Schwarz inequality (1.3) and their generalizations (1.4) and (2.2).

Theorem 2.1. Let $A \in \mathscr{B}(\mathscr{H})$ with the Cartesian decomposition $A=P+i Q$. If $f$ and $g$ are as in Corollary 2.1, then

$$
|\langle A x, y\rangle| \leq\{\|f(|P|) x\|\|g(|P|) y\|+\|f(|Q|) x\|\|g(|Q|) y\|\}
$$

for all $x, y \in \mathscr{H}$.

Proof. Let $P+i Q$ be the Cartesian decomposition of $A$. Setting $B=C=1 \mathscr{H}$ in $(2.2)$, then

$$
\begin{aligned}
|\langle A x, y\rangle| & =\left(\langle P x, y\rangle^{2}+\langle Q x, y\rangle^{2}\right)^{1 / 2} \\
& \leq|\langle P x, y\rangle|+|\langle Q x, y\rangle| \\
& \leq\left\{\|f(|P|) x\|\left\|g\left(\left|P^{*}\right|\right) y\right\|+\|f(|Q|) x\|\left\|g\left(\left|Q^{*}\right|\right) y\right\|\right\}
\end{aligned}
$$


for all $x, y \in \mathscr{H}$, where the last inequality follows form (2.2). So that, the required result follows since $P$ and $Q$ are selfadjoint operators.

Corollary 2.6. Let $A \in \mathscr{B}(\mathscr{H})$ with the Cartesian decomposition $A=P+i Q$. Then,

$$
|\langle A x, y\rangle| \leq\left\{\left\||P|^{\alpha} x\right\|\left\||P|^{(1-\alpha)} y\right\|+\left\||Q|^{\alpha} x\right\|\left\||Q|^{(1-\alpha)} y\right\|\right\}
$$

for all $x, y \in \mathscr{H}$

Proof. Setting $f(t)=t^{\alpha}$ and $g(t)=t^{1-\alpha}, 0 \leq \alpha \leq 1, t \geq 0$ in Theorem 2.1 we get (2.10).

The Cartesian companion decomposition of the Kato's inequality (1.3) can be deduced as follows:

Corollary 2.7. Let $A \in \mathscr{B}(\mathscr{H})$ with the Cartesian decomposition $A=P+i Q$. Then,

$$
\begin{aligned}
|\langle A x, y\rangle| \leq\left\langle|P|^{2 \alpha} x, x\right\rangle^{1 / 2}\left\langle|P|^{2(1-\alpha)} y, y\right\rangle^{1 / 2} & \\
& +\left\langle|Q|^{2 \alpha} x, x\right\rangle^{1 / 2}\left\langle|Q|^{2(1-\alpha)} y, y\right\rangle^{1 / 2}
\end{aligned}
$$

for all $x, y \in \mathscr{H}$ and any $0 \leq \alpha \leq 1$.

Proof. Setting $f(t)=t^{\alpha}$ and $g(t)=t^{1-\alpha}, 0 \leq \alpha \leq 1, t \geq 0$ in Corollary 2.6, then we have

$$
\begin{aligned}
|\langle A x, y\rangle| & \leq\left\||P|^{\alpha} x\right\|\left\||P|^{1-\alpha} y\right\|+\left\||Q|^{\alpha} x\right\|\left\||Q|^{1-\alpha} y\right\| \\
& =\left\langle|P|^{2 \alpha} x, x\right\rangle^{1 / 2}\left\langle|P|^{2(1-\alpha)} y, y\right\rangle^{1 / 2}+\left\langle|Q|^{2 \alpha} x, x\right\rangle^{1 / 2}\left\langle|Q|^{2(1-\alpha)} y, y\right\rangle^{1 / 2}
\end{aligned}
$$

for all $x, y \in \mathscr{H}$, which proves the required result.

Remark 2.2. Some Weyl type inequalities can be deduced by following the same approach considered in [12]. In fact by making use of (2.2) and (2.9) instead of (1.4) in [12], a general Weyl type inequality can be deduced. Similarly, some inequalities for the $p$-Schatten norm can be pointed out following the same pattern in [12]. We shall omit the details.

\section{Numerical Radius inequalities}

In order to prove our results in this section we need some of the following well-known facts.

Lemma 3.1. The Power-Young inequality reads that

$$
a b \leq \frac{a^{\alpha}}{\alpha}+\frac{b^{\beta}}{\beta} \leq\left(\frac{a^{p \alpha}}{\alpha}+\frac{b^{p \beta}}{\beta}\right)^{\frac{1}{p}}
$$

for all $a, b \geq 0$ and $\alpha, \beta>1$ with $\frac{1}{\alpha}+\frac{1}{\beta}=1$ and all $p \geq 1$.

Lemma 3.2. (The McCarty inequality). Let $A \in \mathscr{B}(\mathscr{H})^{+}$, then

$$
\langle A x, x\rangle^{p} \leq\left\langle A^{p} x, x\right\rangle, \quad p \geq 1
$$

for any unit vector $x \in \mathscr{H}$ 
Lemma 3.3. If $A, B \in \mathscr{B}(\mathscr{H})$, then

$$
r(A B) \leq \frac{1}{4}\left(\|A B\|+\|B A\|+\sqrt{(\|A B\|-\|B A\|)^{2}+4 m(A, B)}\right)
$$

where $m(A, B):=\min \{\|A\|\|B A B\|,\|B\|\|A B A\|\}$.

In some of our results we need the following two fundamental norm estimates, which are:

$$
\|A+B\| \leq \frac{1}{2}\left(\|A\|+\|B\|+\sqrt{(\|A\|-\|B\|)^{2}+4\left\|A^{1 / 2} B^{1 / 2}\right\|^{2}}\right),
$$

and

$$
\left\|A^{1 / 2} B^{1 / 2}\right\| \leq\|A B\|^{1 / 2}
$$

Both estimates are valid for all positive operators $A, B \in \mathscr{B}(\mathscr{H})$. Also, it should be noted that (3.4) is sharper than the triangle inequality as pointed out by Kittaneh in [11].

3.1. Inequalities using the mixed Schwarz inequality. Depending on the obtained results in Section 2.1, in this part we provide some numerical radius inequalities. Let us start with the following result.

Theorem 3.1. Let $A, B, C \in \mathscr{B}(\mathscr{H})$ such that $|A| B=B^{*}|A|$ and $\left|A^{*}\right| C=$ $C^{*}\left|A^{*}\right|$. If $f$ and $g$ are nonnegative continuous functions on $[0, \infty)$ satisfying $f(t) g(t)=t(t \geq 0)$, then

$$
\begin{aligned}
w\left(C^{*} A B\right) \leq \frac{1}{2} & r(B) r(C) \cdot\left\|f^{2}(|A|)+g^{2}\left(\left|A^{*}\right|\right)\right\| \\
\leq \frac{1}{16} & \left(\|B\|+\left\|B^{2}\right\|^{1 / 2}\right)\left(\|C\|+\left\|C^{2}\right\|^{1 / 2}\right) \\
& \times\left\{\left\|f^{2}(|A|)\right\|+\left\|g^{2}\left(\left|A^{*}\right|\right)\right\|\right. \\
& \left.+\sqrt{\left(\left\|f^{2}(|A|)\right\|-\left\|g^{2}\left(\left|A^{*}\right|\right)\right\|\right)^{2}+4\left\|f(|A|) g\left(\left|A^{*}\right|\right)\right\|^{2}}\right\}
\end{aligned}
$$

In particular, we have

$$
\begin{aligned}
w\left(C^{*} C\right) \leq & \frac{1}{2} r(C) \cdot\left\|f^{2}(|C|)+g^{2}\left(\left|C^{*}\right|\right)\right\| \\
\leq & \frac{1}{8}\left(\|C\|+\left\|C^{2}\right\|^{1 / 2}\right)\left\{\left\|f^{2}(|C|)\right\|+\left\|g^{2}\left(\left|C^{*}\right|\right)\right\|\right. \\
& \left.\quad+\sqrt{\left(\left\|f^{2}(|C|)\right\|-\left\|g^{2}\left(\left|C^{*}\right|\right)\right\|\right)^{2}+4\left\|f(|C|) g\left(\left|C^{*}\right|\right)\right\|^{2}}\right\}
\end{aligned}
$$


Proof. Setting $y=x$ in (2.2), we get

$$
\begin{aligned}
\left|\left\langle C^{*} A B x, x\right\rangle\right| & \leq r(B) r(C)\|f(|A|) x\|\left\|g\left(\left|A^{*}\right|\right) x\right\| \\
& =r(B) r(C)\left\langle f^{2}(|A|) x, x\right\rangle^{1 / 2}\left\langle g^{2}\left(\left|A^{*}\right|\right) x, x\right\rangle^{1 / 2} \\
& \leq \frac{1}{2} r(B) r(C)\left(\left\langle f^{2}(|A|) x, x\right\rangle+\left\langle g^{2}\left(\left|A^{*}\right|\right) x, x\right\rangle\right) \\
& =\frac{1}{2} r(B) r(C)\left\langle\left(f^{2}(|A|)+g^{2}\left(\left|A^{*}\right|\right)\right) x, x\right\rangle \\
& =\frac{1}{2} r(B) r(C)\left\|\left(f^{2}(|A|)+g^{2}\left(\left|A^{*}\right|\right)\right)\right\| .
\end{aligned}
$$

Thus, by taking the supremum over $x \in \mathscr{H}$ we get the first inequality in (3.6). The second inequality in (3.6) follows by employing (3.3) on the first inequality and use (3.4). The inequality (3.7) follows from (3.6) by setting $B=I$ and $A=C$.

Corollary 3.1. Let $A, B, C \in \mathscr{B}(\mathscr{H})$ such that $|A| B=B^{*}|A|$ and $\left|A^{*}\right| C=$ $C^{*}\left|A^{*}\right|$. Then

$$
\begin{aligned}
w\left(C^{*} A B\right) \leq & \frac{1}{2} r(B) r(C) \cdot\left\||A|^{2 \alpha}+\left|A^{*}\right|^{2(1-\alpha)}\right\| \\
\leq \frac{1}{16} & \left(\|B\|+\left\|B^{2}\right\|^{1 / 2}\right)\left(\|C\|+\left\|C^{2}\right\|^{1 / 2}\right) \\
& \times\left\{\left\||A|^{2 \alpha}\right\|+\left\|\left|A^{*}\right|^{2(1-\alpha)}\right\|\right. \\
& \left.+\sqrt{\left(\left\||A|^{2 \alpha}\right\|-\left\|\left|A^{*}\right|^{2(1-\alpha)}\right\|\right)^{2}+4\left\||A|^{\alpha}\left|A^{*}\right|^{1-\alpha}\right\|^{2}}\right\} .
\end{aligned}
$$

Proof. Setting $f(t)=t^{\alpha}$ and $g(t)=t^{1-\alpha}, 0 \leq \alpha \leq 1, t \geq 0$ in Theorem 3.1 we get (3.8).

Remark 3.1. Setting $\alpha=\frac{1}{2}$ in (3.8) and then employing (3.5) we get

$$
w\left(C^{*} A B\right) \leq \frac{1}{8}\left(\|B\|+\left\|B^{2}\right\|^{1 / 2}\right)\left(\|C\|+\left\|C^{2}\right\|^{1 / 2}\right)\left(\|A\|+\left\|A^{2}\right\|^{1 / 2}\right)
$$

Remark 3.2. Letting $A=C$ and $B=1_{\mathscr{H}}$ in (3.8). Then

$$
\begin{aligned}
w\left(C^{*} C\right) \leq & \frac{1}{2} r(C) \cdot\left\||C|^{2 \alpha}+\left|C^{*}\right|^{2(1-\alpha)}\right\| \\
\leq & \frac{1}{8}\left(\|C\|+\left\|C^{2}\right\|^{1 / 2}\right)\left\{\left\||C|^{2 \alpha}\right\|+\left\|\left|C^{*}\right|^{2(1-\alpha)}\right\|\right. \\
& \left.\quad+\sqrt{\left(\left\||C|^{2 \alpha}\right\|-\left\|\left|C^{*}\right|^{2(1-\alpha)}\right\|\right)^{2}+4\left\||C|^{\alpha}\left|C^{*}\right|^{1-\alpha}\right\|^{2}}\right\} .
\end{aligned}
$$

Moreover, setting $\alpha=\frac{1}{2}$ in the above inequality and use (3.5) with the fact that $\||C|\|=\left\|\left|C^{*}\right|\right\|=\|C\|$. So that we get

$$
w\left(C^{*} C\right) \leq \frac{1}{4}\left(\|C\|+\left\|C^{2}\right\|^{1 / 2}\right)^{2} .
$$


Corollary 3.2. Let $A, B, C \in \mathscr{B}(\mathscr{H})$ such that $|A| C=C^{*}|A|$ and $\left|A^{*}\right| C=$ $C^{*}\left|A^{*}\right|$. If $f$ and $g$ are nonnegative continuous functions on $[0, \infty)$ satisfying $f(t) g(t)=t(t \geq 0)$, then

$$
\begin{aligned}
w\left(C^{*} A C\right) \leq & \frac{1}{2} r^{2}(C) \cdot\left\|f^{2}(|A|)+g^{2}\left(\left|A^{*}\right|\right)\right\| \\
\leq & \frac{1}{16}\left(\|C\|+\left\|C^{2}\right\|^{1 / 2}\right)^{2}\left\{\left\|f^{2}(|A|)\right\|+\left\|g^{2}\left(\left|A^{*}\right|\right)\right\|\right. \\
& \left.\quad+\sqrt{\left(\left\|f^{2}(|A|)\right\|-\left\|g^{2}\left(\left|A^{*}\right|\right)\right\|\right)^{2}+4\left\|f(|A|) g\left(\left|A^{*}\right|\right)\right\|^{2}}\right\}
\end{aligned}
$$

Proof. Setting $B=C$ in Theorem 3.1.

A generalization of Theorem 3.1 to higher order power is given as follows:

Theorem 3.2. Let $A, B \in \mathscr{B}(\mathscr{H})(\Omega)$ such that $|A| B=B^{*}|A|$. If $f, g$ be nonnegative continuous functions on $[0, \infty)$ satisfying $f(t) g(t)=t,(t \geq 0)$, then

$$
w^{p}\left(C^{*} A B\right) \leq r^{p}(B) r^{p}(C) \cdot\left\|\frac{1}{\alpha} f^{\alpha p}(|A|)+\frac{1}{\beta} g^{\beta p}\left(\left|A^{*}\right|\right)\right\|
$$

for all $p \geq 1, \alpha \geq \beta>1$ with $\frac{1}{\alpha}+\frac{1}{\beta}=1$ and $\beta p \geq 2$. Moreover we have

$$
\begin{aligned}
w^{p}\left(C^{*} A B\right) \leq & \frac{1}{2^{p+2}} \cdot \gamma \cdot\left(\|B\|+\left\|B^{2}\right\|^{1 / 2}\right)^{p}\left(\|C\|+\left\|C^{2}\right\|^{1 / 2}\right)^{p} \\
& \times\left\{\left\|f^{\alpha p}(|A|)\right\|+\left\|g^{\beta p}\left(\left|A^{*}\right|\right)\right\|\right. \\
& \left.+\sqrt{\left[\left|f^{\alpha p}(|A|)\right|-\left\|g^{\beta p}\left(\left|A^{*}\right|\right)\right\|\right]^{2}+4\left\|f^{p \alpha}(|A|) g^{p \beta}\left(\left|A^{*}\right|\right)\right\|^{2}}\right\}
\end{aligned}
$$

where $\gamma=\max \left\{\frac{1}{\alpha}, \frac{1}{\beta}\right\}$.

Proof. Setting $u=x$ in the generalized mixed Schwarz inequality (2.2), we have

$$
\begin{aligned}
& \left|\left\langle C^{*} A B x, x\right\rangle\right|^{p} \\
& \leq r^{p}(B) r^{p}(C)\|f(|A|) x\|^{p}\left\|g\left(\left|A^{*}\right|\right) x\right\|^{p} \\
& =r^{p}(B) r^{p}(C)\left\langle f^{2}(|A|) x, x\right\rangle^{\frac{p}{2}}\left\langle g^{2}\left(\left|A^{*}\right|\right) x, x\right\rangle^{\frac{p}{2}} \\
& \leq r^{p}(B) r^{p}(C)\left[\frac{1}{\alpha}\left\langle f^{2}(|A|) x, x\right\rangle^{\frac{\alpha p}{2}}+\frac{1}{\beta}\left\langle g^{2}\left(\left|A^{*}\right|\right) x, x\right\rangle^{\frac{\beta p}{2}}\right] \\
& \leq r^{p}(B) r^{p}(C)\left[\frac{1}{\alpha}\left\langle f^{\alpha p}(|A|) x, x\right\rangle+\frac{1}{\beta}\left\langle g^{\beta p}\left(\left|A^{*}\right|\right) x, x\right\rangle\right] \\
& =r^{p}(B) r^{p}(C)\left\langle\left[\frac{1}{\alpha} f^{\alpha p}(|A|)+\frac{1}{\beta} g^{\beta p}\left(\left|A^{*}\right|\right)\right] x, x\right\rangle .
\end{aligned}
$$


Taking the supremum over $x \in \mathscr{H}$, we obtain the inequality in (3.10). To obtain the second inequality, by (3.4) we have

$$
\begin{aligned}
& \left\|\frac{1}{\alpha} f^{\alpha p}(|A|)+\frac{1}{\beta} g^{\beta p}\left(\left|A^{*}\right|\right)\right\| \\
& \leq \max \left\{\frac{1}{\alpha}, \frac{1}{\beta}\right\} \cdot\left\|f^{\alpha p}(|A|)+g^{\beta p}\left(\left|A^{*}\right|\right)\right\| \\
& \leq \frac{1}{2} \gamma\left(\left\|f^{\alpha p}(|A|)\right\|+\left\|g^{\beta p}\left(\left|A^{*}\right|\right)\right\|\right. \\
& \left.\quad+\sqrt{\left[\left|f^{\alpha p}(|A|)\right|-\left\|g^{\beta p}\left(\left|A^{*}\right|\right)\right\|\right]^{2}+4\left\|f^{p \alpha}(|A|) g^{p \beta}\left(\left|A^{*}\right|\right)\right\|^{2}}\right) .
\end{aligned}
$$

Now, employing (3.3) with $B=1_{\mathscr{H}}$ and then substituting all in (3.10) we get (3.11).

Remark 3.3. Letting $u=x$ in (2.7), then by taking the supremum over $x \in \mathscr{H}$ with $\|x\|=1$ so that we get

$$
\begin{aligned}
& w\left(\sum_{i=1}^{n} C_{i}^{*} A_{i} B_{i}\right) \\
& \leq \sum_{i=1}^{n} r\left(B_{i}\right) r\left(C_{i}\right)\left\|f\left(\left|A_{i}\right|\right)\right\|\left\|g\left(\left|A_{i}^{*}\right|\right)\right\| \\
& \leq \max _{1 \leq i \leq n}\left\{r\left(B_{i}\right) r\left(C_{i}\right)\right\} \cdot\left(\sum_{i=1}^{n}\left\|f\left(\left|A_{i}\right|\right)\right\|^{p}\right)^{1 / p}\left(\sum_{i=1}^{n}\left\|g\left(\left|A_{i}^{*}\right|\right)\right\|^{q}\right)^{1 / q} .
\end{aligned}
$$

Following the same approach applied in the proof of Theorems 3.1 and 3.2, one can state other bounds for the second inequality above. Several special cases can also be obtained as in the Corollaries 3.1 and 3.2. Of course the same inequalities still valid for norms instead of numerical radius.

\subsection{Inequalities using the mixed hybrid Schwarz inequality.}

Theorem 3.3. Let $A \in \mathscr{B}(\mathscr{H})$ with the Cartesian decomposition $A=P+i Q$. If $f$ and $g$ are as in Corollary 2.1. Then

$$
w(A) \leq\left\|f^{p}(|P|)+f^{p}(|Q|)\right\|^{1 / p}\left\|g^{q}(|P|)+g^{q}(|Q|)\right\|^{1 / q}
$$

for all $p, q \geq 2$ with $\frac{1}{p}+\frac{1}{q}=1$. 
Proof. Letting $y=x$ in (2.8), then we have

$$
\begin{aligned}
& |\langle A x, y\rangle| \\
& \leq\{\|f(|P|) x\|\|g(|P|) y\|+\|f(|Q|) x\|\|g(|Q|) y\|\} \\
& \leq\left(\|f(|P|) x\|^{p}+\|f(|Q|) x\|^{p}\right)^{1 / p} \\
& \quad \times\left(\|g(|P|) y\|^{q}+\|g(|Q|) y\|^{q}\right)^{1 / q} \quad \text { (by Hölder inequaity) } \\
& \leq\left(\left\langle f^{2}(|P|) x, x\right\rangle^{p / 2}+\left\langle f^{2}(|Q|) x, x\right\rangle^{p / 2}\right)^{1 / p} \\
& \quad \times\left(\left\langle g^{2}(|P|) x, x\right\rangle^{q / 2}+\left\langle g^{2}(|Q|) x, x\right\rangle^{q / 2}\right)^{1 / q} \\
& \left.\leq\left(\left\langle f^{p}(|P|) x, x\right\rangle+\left\langle f^{p}(|Q|) x, x\right\rangle\right)^{1 / p} \quad \quad \times\left(\left\langle g^{q}(|P|) x, x\right\rangle+\left\langle g^{q}(|Q|) x, x\right\rangle\right)^{1 / q} \quad \quad \text { by }(3.2)\right) \\
& \quad\left\langle\left[f^{p}(|P|)+f^{p}(|Q|)\right] x, x\right\rangle^{1 / p}\left\langle\left[g^{q}(|P|)+g^{q}(|Q|)\right] x, x\right\rangle^{1 / q}
\end{aligned}
$$

for all $p, q \geq 2$ with $\frac{1}{p}+\frac{1}{q}=1$. Taking the supremum over all unit vector $x \in \mathscr{H}$ we get the desired result.

However, we can still have a little more manipulation; by employing (3.4) for the above two norms we get

$$
\begin{aligned}
& \left\|\left(f^{p}(|P|)+f^{p}(|Q|)\right)\right\| \\
& \leq \frac{1}{2}\left(\left\|f^{p}(|P|)\right\|+\left\|f^{p}(|Q|)\right\|\right. \\
& \left.\quad+\sqrt{\left(\left\|f^{p}(|P|)\right\|-\left\|f^{p}(|Q|)\right\|\right)^{2}+4\left\|f^{p / 2}(|P|) f^{p / 2}(|Q|)\right\|^{2}}\right),
\end{aligned}
$$

and

$$
\begin{aligned}
& \left\|\left(g^{q}(|P|)+g^{q}(|Q|)\right)\right\| \\
& \leq \frac{1}{2}\left(\left\|g^{q}(|P|)\right\|+\left\|g^{q}(|Q|)\right\|\right. \\
& \left.\quad+\sqrt{\left(\left\|g^{q}(|P|)\right\|-\left\|g^{q}(|Q|)\right\|\right)^{2}+4\left\|g^{q / 2}(|P|) g^{q / 2}(|Q|)\right\|^{2}}\right) .
\end{aligned}
$$

Substituting (3.14) and (3.15) in (3.13) we get another refinement of (3.13).

Remark 3.4. In an interesting case, one may consider $f(t)=t^{\alpha}$ and $g(t)=t^{1-\alpha}$, $\alpha \in[0,1]$ and $p=q=2$. If we wish for $\alpha=\frac{1}{2}$, after some manipulations and making of use (3.5) we get

$$
w(A) \leq \frac{1}{2} \cdot\left(\||P|\|+\||Q|\|+\sqrt{(\||P|\|-\||Q|\|)^{2}+4\||P||Q|\|}\right) .
$$

It should be noted that the authors in [3] have shown that $w(A) \leq\||P|\|+\||Q|\|$, it is not hard to show that our estimate is better than the previous one.

Remark 3.5. Following the same approach considered in the proof of Theorem 3.2 , one may state another bound of (3.13). 


\section{Acknowledgements}

The author wishes to thank the referee for his fruitful comments and careful reading of the original manuscript of this work, which have improved the final version.

\section{References}

[1] S.S. Dragomir, Inequalities for the numerical radius of linear operators in Hilbert spaces, SpringerBriefs in Mathematics, 2013.

[2] S.S. Dragomir, Some inequalities for the norm and the numerical radius of linear operator in Hilbert spaces, Tamkang J. Math., 39 (1) (2008), 1-7.

[3] M. El-Haddad and F. Kittaneh, Numerical radius inequalities for Hilbert space operators. II. Studia Math., 182 (2) (2007), 133-140.

[4] K.E. Gustafson and D.K. Rao, Numerical Range: The Field of Values of Linear Operators and Matrices, Springer-Verlag New York, 1996.

[5] P.R. Halmos, A Hilbert space problem book, Van Nostrand Company, Inc., Princeton, N.J., 1967.

[6] R.A. Horn and C.R. Johnson, Topics in Matrix Analysis, Cambridge University Press, Cambridge, 1991.

[7] F. Kittaneh, Numerical radius inequalities for Hilbert space operators, Studia Math., 168 (1) (2005), 73-80.

[8] F. Kittaneh, Spectral radius inequalities for Hilbert space operators, Proc. Amer. Math. Soc., 134 (2) (2005), 385-390.

[9] F. Kittaneh, A numerical radius inequality and an estimate for the numerical radius of the Frobenius companion matrix, Studia Math., 158 (2003), 11-17.

[10] F. Kittaneh, Bounds for the zeros of polynomials from matrix inequalities, Arch.Math., (Basel) 81 (2003), 601-608.

[11] F. Kittaneh, Norm inequalities for sums of positive operators, J. Operator Theory, 48 (2002), 95-103.

[12] F. Kittaneh, Notes on some inequalities for Hilbert Space operators, Publ. Res. Inst. Math. Sci., 24 (2) (1988), 283-293.

[13] T. Kato, Notes on some inequalities for linear operators, Math. Ann., 125 (1952), $208-212$.

[14] C.-S. Lin and S.S. Dragomir, On High-power Operator inequalities and spectral radii of operators, Publ. RIMS, Kyoto Univ., 42 (2006), 391-397.

[15] M.S. Moslehian, M. Sattari and K. Shebrawi, Extensions of Euclidean operator radius inequalities, Mathematica Scandinavica, 120 (1) (2017), 129-144.

[16] W. Reid, Symmetrizable completely continuous linear tarnsformations in Hilbert space, Duke Math., 18 (1951), 41-56.

[17] M. Sattari, M.S. Moslehian and T. Yamazaki, Some genaralized numerical radius inequalities for Hilbert space operators, Linear Algebra Appl., 470 (2014), 1-12.

[18] T. Yamazaki, On upper and lower bounds of the numerical radius and an equality condition, Studia Math., 178 (2007), 83-89.

Mohammad W. Alomari

Department of Mathematics, Faculty of Science and Information Technology, Irbid National University, P.O. Box 2600, Irbid, P.C. 21110, Jordan.

E-mail address: mwomath@gmail.com

Received: April 11, 2019; Revised: October 3, 2019; Accepted: October 18, 2019 